\title{
Sharp bounds for Neuman means in terms of one-parameter family of bivariate means
}

\author{
Zhi-Hua Shao', Wei-Mao Qian² and Yu-Ming Chu*
}

"Correspondence:

chuyuming2005@126.com

'School of Mathematics and

Computation Sciences, Hunan City

University, Yiyang, 413000, China

Full list of author information is

available at the end of the article

\begin{abstract}
We present the best possible parameters $p_{1}, p_{2}, p_{3}, p_{4}, q_{1}, q_{2}, q_{3}, q_{4} \in[0,1]$ such that the double inequalities $G_{p_{1}}(a, b)<S_{H A}(a, b)<G_{q_{1}}(a, b), Q_{p_{2}}(a, b)<S_{C A}(a, b)<Q_{q_{2}}(a, b)$, $H_{p_{3}}(a, b)<S_{A H}(a, b)<H_{a_{3}}(a, b), C_{p_{4}}(a, b)<S_{A C}(a, b)<C_{q_{4}}(a, b)$ hold for all $a, b>0$ with $a \neq b$, where $S_{H A}, S_{C A}, S_{A H}, S_{A C}$ are the Neuman means, and $G_{p}, Q_{p}, H_{p}, C_{p}$ are the one-parameter means.
\end{abstract}

MSC: $26 \mathrm{E} 60$

Keywords: Neuman means; one-parameter mean; harmonic mean; geometric mean; arithmetic mean; quadratic mean; contraharmonic mean

\section{Introduction}

Let $a, b>0$ with $a \neq b$. Then the Schwab-Borchardt mean $S B(a, b)[1-3]$, and the Neuman means $S_{H A}(a, b), S_{A H}(a, b), S_{C A}(a, b)$, and $S_{A C}(a, b)[4,5]$ of $a$ and $b$ are given by

$$
\begin{array}{ll}
S B(a, b)=\frac{\sqrt{b^{2}-a^{2}}}{\cos ^{-1}(a / b)} \quad(a<b), & S B(a, b)=\frac{\sqrt{a^{2}-b^{2}}}{\cosh ^{-1}(a / b)} \quad(a>b), \\
S_{H A}(a, b)=S B[H(a, b), A(a, b)], & S_{A H}(a, b)=S B[A(a, b), H(a, b)], \\
S_{C A}(a, b)=S B[C(a, b), A(a, b)], & S_{A C}(a, b)=S B[A(a, b), C(a, b)],
\end{array}
$$

respectively. Here, $\cos ^{-1}(x)$ and $\cosh ^{-1}(x)=\log \left(x+\sqrt{x^{2}-1}\right)$ are, respectively, the inverse cosine and inverse hyperbolic cosine functions, and $H(a, b)=2 a b /(a+b), A(a, b)=(a+$ $b) / 2$, and $C(a, b)=\left(a^{2}+b^{2}\right) /(a+b)$ are, respectively, the classical harmonic, arithmetic, and contraharmonic means of $a$ and $b$.

Let $v=(a-b) /(a+b) \in(-1,1)$, and $p \in(0, \infty), q \in(0, \pi / 2), r \in(0, \log (2+\sqrt{3}))$, and $s \in$ $(0, \pi / 3)$ be the parameters such that $1 / \cosh (p)=\cos (q)=1-v^{2}$ and $\cosh (r)=1 / \cosh (s)=$ $1+v^{2}$. Then the following explicit formulas were found by Neuman [4]:

$$
\begin{array}{ll}
S_{A H}(a, b)=A(a, b) \frac{\tanh (p)}{p}, & S_{H A}(a, b)=A(a, b) \frac{\sin (q)}{q}, \\
S_{C A}(a, b)=A(a, b) \frac{\sinh (r)}{r}, & S_{A C}(a, b)=A(a, b) \frac{\tan (s)}{s} .
\end{array}
$$

O2014 Shao et al.; licensee Springer. This is an Open Access article distributed under the terms of the Creative Commons Attribution License (http://creativecommons.org/licenses/by/2.0), which permits unrestricted use, distribution, and reproduction in any medium, provided the original work is properly cited. 
Let $p \in[0,1]$ and $N$ be a bivariate symmetric mean. Then the one-parameter bivariate mean $N_{p}(a, b)$ was defined by Neuman [6] as follows:

$$
N_{p}(a, b)=N\left[\frac{(1+p)}{2} a+\frac{(1-p)}{2} b, \frac{(1+p)}{2} b+\frac{(1-p)}{2} a\right] .
$$

Recently, the Neuman means $S_{A H}, S_{H A}, S_{C A}$, and $S_{A C}$, and the one-parameter bivariate mean $N_{p}$ have been the subject of intensive research. He et al. [7] found the greatest values $\alpha_{1}, \alpha_{2} \in[0,1 / 2]$, and $\alpha_{3}, \alpha_{4} \in[1 / 2,1]$, and the least values $\beta_{1}, \beta_{2} \in[0,1 / 2]$, and $\beta_{3}, \beta_{4} \in[1 / 2,1]$ such that the double inequalities

$$
\begin{aligned}
& H\left[\alpha_{1} a+\left(1-\alpha_{1}\right) b, \alpha_{1} b+\left(1-\alpha_{1}\right) a\right]<S_{A H}(a, b)<H\left[\beta_{1} a+\left(1-\beta_{1}\right) b, \beta_{1} b+\left(1-\beta_{1}\right) a\right], \\
& H\left[\alpha_{2} a+\left(1-\alpha_{2}\right) b, \alpha_{2} b+\left(1-\alpha_{2}\right) a\right]<S_{H A}(a, b)<H\left[\beta_{2} a+\left(1-\beta_{2}\right) b, \beta_{2} b+\left(1-\beta_{2}\right) a\right], \\
& C\left[\alpha_{3} a+\left(1-\alpha_{3}\right) b, \alpha_{3} b+\left(1-\alpha_{3}\right) a\right]<S_{C A}(a, b)<C\left[\beta_{3} a+\left(1-\beta_{3}\right) b, \beta_{3} b+\left(1-\beta_{3}\right) a\right], \\
& C\left[\alpha_{4} a+\left(1-\alpha_{4}\right) b, \alpha_{4} b+\left(1-\alpha_{4}\right) a\right]<S_{A C}(a, b)<C\left[\beta_{4} a+\left(1-\beta_{4}\right) b, \beta_{4} b+\left(1-\beta_{4}\right) a\right]
\end{aligned}
$$

hold for all $a, b>0$ with $a \neq b$.

In $[4,5]$, Neuman proved that the inequalities

$$
\begin{aligned}
& H(a, b)<S_{A H}(a, b)<L(a, b)<S_{H A}(a, b)<P(a, b), \\
& T(a, b)<S_{C A}(a, b)<Q(a, b)<S_{A C}(a, b)<C(a, b), \\
& H^{1 / 3}(a, b) A^{2 / 3}(a, b)<S_{H A}(a, b)<\frac{1}{3} H(a, b)+\frac{2}{3} A(a, b), \\
& C^{1 / 3}(a, b) A^{2 / 3}(a, b)<S_{C A}(a, b)<\frac{1}{3} C(a, b)+\frac{2}{3} A(a, b), \\
& A^{1 / 3}(a, b) H^{2 / 3}(a, b)<S_{A H}(a, b)<\frac{1}{3} A(a, b)+\frac{2}{3} H(a, b), \\
& A^{1 / 3}(a, b) C^{2 / 3}(a, b)<S_{A C}(a, b)<\frac{1}{3} A(a, b)+\frac{2}{3} C(a, b)
\end{aligned}
$$

hold for all $a, b>0$ with $a \neq b$, where $L(a, b)=(a-b) /(\log a-\log b), P(a, b)=(a-$ $b) /[2 \arcsin ((a-b) /(a+b))], Q(a, b)=\sqrt{\left(a^{2}+b^{2}\right) / 2}$, and $T(a, b)=(a-b) /[2 \arctan ((a-$ $b) /(a+b))]$ are, respectively, the logarithmic, first Seiffert, quadratic, and second Seiffert means of $a$ and $b$.

Qian and Chu [8] proved that the double inequalities

$$
\begin{aligned}
& \alpha_{1} A(a, b)+\left(1-\alpha_{1}\right) G(a, b)<S_{H A}(a, b)<\beta_{1} A(a, b)+\left(1-\beta_{1}\right) G(a, b), \\
& \alpha_{2} A(a, b)+\left(1-\alpha_{2}\right) Q(a, b)<S_{C A}(a, b)<\beta_{2} A(a, b)+\left(1-\beta_{2}\right) Q(a, b)
\end{aligned}
$$

hold for all $a, b>0$ with $a \neq b$ if and only if $\alpha_{1} \leq 1 / 3, \beta_{1} \geq \pi / 2, \alpha_{2} \geq 1 / 3$, and $\beta_{2} \leq$ $[\sqrt{2} \log (2+\sqrt{3})-\sqrt{3}] /[(\sqrt{2}-1) \log (2+\sqrt{3})]=0.2394 \cdots$, where $G(a, b)=\sqrt{a b}$ is the geometric mean of $a$ and $b$. 
In [9], the authors proved that the double inequalities

$$
\begin{aligned}
& \alpha_{1} {\left[\frac{H(a, b)}{3}+\frac{2 A(a, b)}{3}\right]+\left(1-\alpha_{1}\right) H^{1 / 3}(a, b) A^{2 / 3}(a, b)<S_{H A}(a, b) } \\
&<\beta_{1}\left[\frac{H(a, b)}{3}+\frac{2 A(a, b)}{3}\right]+\left(1-\beta_{1}\right) H^{1 / 3}(a, b) A^{2 / 3}(a, b), \\
& \alpha_{2}\left[\frac{C(a, b)}{3}+\frac{2 A(a, b)}{3}\right]+\left(1-\alpha_{2}\right) C^{1 / 3}(a, b) A^{2 / 3}(a, b)<S_{C A}(a, b) \\
& \quad<\beta_{2}\left[\frac{C(a, b)}{3}+\frac{2 A(a, b)}{3}\right]+\left(1-\beta_{2}\right) C^{1 / 3}(a, b) A^{2 / 3}(a, b), \\
& \alpha_{3}\left[\frac{A(a, b)}{3}+\frac{2 H(a, b)}{3}\right]+\left(1-\alpha_{3}\right) A^{1 / 3}(a, b) H^{2 / 3}(a, b)<S_{A H}(a, b) \\
& \quad<\beta_{3}\left[\frac{A(a, b)}{3}+\frac{2 H(a, b)}{3}\right]+\left(1-\beta_{3}\right) A^{1 / 3}(a, b) H^{2 / 3}(a, b), \\
& \alpha_{4}\left[\frac{A(a, b)}{3}+\frac{2 C(a, b)}{3}\right]+\left(1-\alpha_{4}\right) A^{1 / 3}(a, b) C^{2 / 3}(a, b)<S_{A C}(a, b) \\
& \quad<\beta_{4}\left[\frac{A(a, b)}{3}+\frac{2 C(a, b)}{3}\right]+\left(1-\beta_{4}\right) A^{1 / 3}(a, b) C^{2 / 3}(a, b)
\end{aligned}
$$

hold for all $a, b>0$ with $a \neq b$ if and only if $\alpha_{1} \leq 4 / 5, \beta_{1} \geq 3 / \pi, \alpha_{2} \leq 3[\sqrt[3]{2} \log (2+\sqrt{3})-$ $\sqrt{3}] /[(3 \sqrt[3]{2}-4) \log (2+\sqrt{3})]=0.7528 \cdots, \beta_{2} \geq 4 / 5, \alpha_{3} \leq 0, \beta_{3} \geq 4 / 5, \alpha_{4} \leq 4 / 5$, and $\beta_{4} \geq$ $3(3 \sqrt{3}-\sqrt[3]{4} \pi) /[(5-3 \sqrt[3]{4}) \pi]=0.8400 \cdots$.

Let $p, p_{i}, q_{i}, \alpha_{j}, \beta_{j} \in[0,1](i, j=1,2, \ldots, 8)$. Then Neuman $[6,10]$ proved that the inequalities

$$
\begin{aligned}
& H_{p_{1}}(a, b)<P(a, b)<H_{q_{1}}(a, b), \quad G_{p_{2}}(a, b)<P(a, b)<G_{q_{2}}(a, b), \\
& Q_{p_{3}}(a, b)<T(a, b)<Q_{q_{3}}(a, b), \quad C_{p_{4}}(a, b)<T(a, b)<C_{q_{4}}(a, b), \\
& Q_{p_{5}}(a, b)<M(a, b)<Q_{q_{5}}(a, b), \quad C_{p_{6}}(a, b)<M(a, b)<C_{q_{6}}(a, b), \\
& H_{p_{7}}(a, b)<L(a, b)<H_{q_{7}}(a, b), \quad G_{p_{8}}(a, b)<L(a, b)<G_{q_{8}}(a, b), \\
& \alpha_{1} A(a, b)+\left(1-\alpha_{1}\right) G_{p}(a, b)<P_{p}(a, b)<\beta_{1} A(a, b)+\left(1-\beta_{1}\right) G_{p}(a, b), \\
& \alpha_{2} Q_{p}(a, b)+\left(1-\alpha_{2}\right) A(a, b)<T_{p}(a, b)<\beta_{2} Q_{p}(a, b)+\left(1-\beta_{2}\right) A(a, b), \\
& \alpha_{3} Q_{p}(a, b)+\left(1-\alpha_{3}\right) A(a, b)<M_{p}(a, b)<\beta_{3} Q_{p}(a, b)+\left(1-\beta_{3}\right) A(a, b) \\
& \alpha_{4} A(a, b)+\left(1-\alpha_{4}\right) G_{p}(a, b)<L_{p}(a, b)<\beta_{4} A(a, b)+\left(1-\beta_{4}\right) G_{p}(a, b), \\
& A^{\alpha_{5}}(a, b) G_{p}^{1-\alpha_{5}}(a, b)<P_{p}(a, b)<A^{\beta_{5}}(a, b) G_{p}^{1-\beta_{5}}(a, b) \\
& Q_{p}^{\alpha_{6}}(a, b) A^{1-\alpha_{6}}(a, b)<T_{p}(a, b)<Q_{p}^{\beta_{6}}(a, b) A^{1-\beta_{6}}(a, b) \\
& Q_{p}^{\alpha_{7}}(a, b) A^{1-\alpha_{7}}(a, b)<M_{p}(a, b)<Q_{p}^{\beta_{7}}(a, b) A^{1-\beta_{7}}(a, b) \\
& A^{\alpha_{8}}(a, b) G_{p}^{1-\alpha_{8}}(a, b)<L_{p}(a, b)<A^{\beta_{8}}(a, b) G_{p}^{1-\beta_{8}}(a, b)
\end{aligned}
$$

hold for all $a, b>0$ with $a \neq b$ if and only if $p_{1} \geq \sqrt{1-2 / \pi}, q_{1} \leq \sqrt{6} / 6, p_{2} \geq \sqrt{1-4 / \pi^{2}}, q_{2} \leq$ $\sqrt{3} / 3, p_{3} \leq \sqrt{16 / \pi^{2}-1}, q_{3} \geq \sqrt{6} / 3, p_{4} \leq \sqrt{4 / \pi-1}, q_{4} \geq \sqrt{3} / 3, p_{5} \leq \sqrt{1 / \log ^{2}(1+\sqrt{2})-1}$, 
$q_{5} \geq \sqrt{3} / 3, p_{6} \leq \sqrt{1 / \log (1+\sqrt{2})-1}, q_{6} \geq \sqrt{6} / 6, p_{7}=1, q_{7} \leq \sqrt{3} / 3, p_{8}=1, q_{8} \leq \sqrt{6} / 3$, $\alpha_{1} \leq 2 / \pi, \beta_{1} \geq 2 / 3, \alpha_{2} \leq(4-\pi) /[(\sqrt{2}-1) \pi], \beta_{2} \geq 2 / 3, \alpha_{3} \leq[1-\log (1+\sqrt{2})] /[(\sqrt{2}-$ 1) $\log (1+\sqrt{2})], \beta_{3} \geq 1 / 3, \alpha_{4}=0, \beta_{4} \geq 1 / 3, \alpha_{5} \leq 2 / 3, \beta_{5}=1, \alpha_{6} \leq 2 / 3, \beta_{6} \geq(4 \log 2-$ $2 \log \pi) / \log 2, \alpha_{7} \leq 1 / 3, \beta_{7} \geq-\log [\log (1+\sqrt{2})] / \log [\cosh (\log (1+\sqrt{2}))], \alpha_{8} \leq 1 / 3, \beta_{8}=1$, where $M(a, b)=(a-b) /\left[2 \sinh ^{-1}((a-b) /(a+b))\right]$ is the Neuman-Sándor mean of $a$ and $b$.

The main purpose of this paper is to present the best possible parameters $p_{1}, p_{2}, p_{3}, p_{4}$, $q_{1}, q_{2}, q_{3}, q_{4}$ on the interval $[0,1]$ such that the double inequalities

$$
\begin{array}{ll}
G_{p_{1}}(a, b)<S_{H A}(a, b)<G_{q_{1}}(a, b), & Q_{p_{2}}(a, b)<S_{C A}(a, b)<Q_{q_{2}}(a, b), \\
H_{p_{3}}(a, b)<S_{A H}(a, b)<H_{q_{3}}(a, b), & C_{p_{4}}(a, b)<S_{A C}(a, b)<C_{q_{4}}(a, b)
\end{array}
$$

hold for all $a, b>0$ with $a \neq b$.

\section{Main results}

Theorem 2.1 Let $p_{1}, q_{1} \in[0,1]$. Then the double inequality

$$
G_{p_{1}}(a, b)<S_{H A}(a, b)<G_{q_{1}}(a, b)
$$

holds for all $a, b>0$ with $a \neq b$ if and only if $p_{1} \geq \sqrt{6} / 3$ and $q_{1} \leq \sqrt{1-4 / \pi^{2}}$.

Proof Without loss of generality, we assume that $a>b$. Let $v=(a-b) /(a+b), \lambda=v \sqrt{2-v^{2}}$, $x=\sqrt{1-\lambda^{2}}$ and $p \in[0,1]$. Then $v, \lambda, x \in(0,1)$, and (1.1) and (1.3) lead to

$$
\begin{aligned}
S_{H A}(a, b)-G_{p}(a, b) & =A(a, b)\left[\frac{\lambda}{\arcsin (\lambda)}-\sqrt{1-p^{2}\left(1-\sqrt{1-\lambda^{2}}\right)}\right] \\
& =\frac{A(a, b) \sqrt{1-p^{2}\left(1-\sqrt{1-\lambda^{2}}\right)}}{\arcsin (\lambda)} F(x),
\end{aligned}
$$

where

$$
\begin{aligned}
& F(x)=\frac{\sqrt{1-x^{2}}}{\sqrt{1-p^{2}(1-x)}}-\arcsin \left(\sqrt{1-x^{2}}\right), \\
& F(0)=\frac{1}{\sqrt{1-p^{2}}}-\frac{\pi}{2}, \quad F(1)=0, \\
& F^{\prime}(x)=-\frac{(1-x) f(x)}{2 \sqrt{1-x^{2}}\left(p^{2} x+1-p^{2}\right)^{3 / 2}\left[2\left(p^{2} x+1-p^{2}\right)^{3 / 2}+p^{2} x+2\left(1-p^{2}\right) x+p^{2}\right]},
\end{aligned}
$$

where

$$
\begin{aligned}
f(x)= & -p^{4} x^{3}+\left(4 p^{6}+3 p^{4}-4 p^{2}\right) x^{2} \\
& +\left(-8 p^{6}+9 p^{4}+4 p^{2}-4\right) x+\left(4 p^{6}-11 p^{4}+12 p^{2}-4\right) \\
f^{\prime}(x)= & -3 p^{4} x^{2}+2\left(4 p^{6}+3 p^{4}-4 p^{2}\right) x+\left(-8 p^{6}+9 p^{4}+4 p^{2}-4\right) .
\end{aligned}
$$

We divide the discussion into two cases. 
Case $1 p=\sqrt{6} / 3$. Then (2.6) becomes

$$
f(x)=\frac{4}{27}(1-x)\left(3 x^{2}+4 x+2\right)
$$

From (2.5) and (2.8) we clearly see that $F(x)$ is strictly decreasing on $[0,1]$, then $(2.4)$ leads to the conclusion that

$$
F(x)>0
$$

for all $x \in(0,1)$.

Therefore,

$$
S_{H A}(a, b)>G_{\sqrt{6} / 3}(a, b)
$$

for all $a, b>0$ with $a \neq b$ follows from (2.2) and (2.9).

Case $2 p=\sqrt{1-4 / \pi^{2}}$. Then numerical computations lead to

$$
\begin{aligned}
& 4 p^{6}+3 p^{4}-4 p^{2}=\frac{3 \pi^{6}-56 \pi^{4}+240 \pi^{2}-256}{\pi^{6}}<0, \\
& -8 p^{6}+9 p^{4}+4 p^{2}-4=\frac{\pi^{6}+8 \pi^{4}-240 \pi^{2}+512}{\pi^{6}}<0, \\
& f(0)=4 p^{6}-11 p^{4}+12 p^{2}-4=\frac{\pi^{6}-8 \pi^{4}+16 \pi^{2}-256}{\pi^{6}}>0, \\
& f(1)=4\left(3 p^{2}-2\right)=-\frac{4\left(12-\pi^{2}\right)}{\pi^{2}}<0 .
\end{aligned}
$$

It follows from (2.7) and (2.11) together with (2.12) that $f(x)$ is strictly decreasing on $[0,1]$. Then inequalities (2.13) and (2.14) together with (2.5) lead to the conclusion that there exists $\lambda_{1} \in(0,1)$ such that $F(x)$ is strictly decreasing on $\left[0, \lambda_{1}\right]$ and strictly increasing on $\left[\lambda_{1}, 1\right]$.

Note that inequality (2.4) becomes

$$
F(0)=F(1)=0 .
$$

From (2.2), (2.15), and the piecewise monotonicity of $F(x)$ we clearly see that the inequality

$$
S_{H A}(a, b)<G_{\sqrt{1-4 / \pi^{2}}}(a, b)
$$

holds for all $a, b>0$ with $a \neq b$.

Note that

$$
\begin{aligned}
& \lim _{\lambda \rightarrow 0^{+}} \frac{\sqrt{\arcsin ^{2}(\lambda)-\lambda^{2}}}{\arcsin (\lambda) \sqrt{1-\sqrt{1-\lambda^{2}}}}=\frac{\sqrt{6}}{3}, \\
& \lim _{\lambda \rightarrow 1} \frac{\sqrt{\arcsin ^{2}(\lambda)-\lambda^{2}}}{\arcsin (\lambda) \sqrt{1-\sqrt{1-\lambda^{2}}}}=\sqrt{1-\frac{4}{\pi^{2}}} .
\end{aligned}
$$


Therefore, Theorem 2.1 follows from (2.10) and (2.16)-(2.18) together with the fact that inequality (2.1) is equivalent to the inequality (2.19) as follows:

$$
q_{1}<\frac{\sqrt{\arcsin ^{2}(\lambda)-\lambda^{2}}}{\arcsin (\lambda) \sqrt{1-\sqrt{1-\lambda^{2}}}}<p_{1} .
$$

Theorem 2.2 Let $p_{2}, q_{2} \in[0,1]$. Then the double inequality

$$
Q_{p_{2}}(a, b)<S_{C A}(a, b)<Q_{q_{2}}(a, b)
$$

holds for all $a, b>0$ with $a \neq b$ if and only if $p_{2} \leq \sqrt{6} / 3$ and $q_{2} \geq \sqrt{3 / \log ^{2}(2+\sqrt{3})-1}=$ $0.8542 \cdots$.

Proof Without loss of generality, we assume that $a>b$. Let $v=(a-b) /(a+b), \mu=v \sqrt{2+v^{2}}$, $x=\sqrt{1+\mu^{2}}$, and $p \in[0,1]$. Then $v \in(0,1), \mu \in(0, \sqrt{3}), x \in(1,2)$, and (1.2) and (1.3) lead to

$$
\begin{aligned}
S_{C A}(a, b)-Q_{p}(a, b) & =A(a, b)\left[\frac{\mu}{\sinh ^{-1}(\mu)}-\sqrt{1+p^{2}\left(\sqrt{1+\mu^{2}}-1\right)}\right] \\
& =\frac{A(a, b) \sqrt{1+p^{2}\left(\sqrt{1+\mu^{2}}-1\right)}}{\sinh ^{-1}(\mu)} G(x),
\end{aligned}
$$

where

$$
\begin{aligned}
& G(x)=\frac{\sqrt{x^{2}-1}}{\sqrt{1+p^{2}(x-1)}}-\sinh ^{-1}\left(\sqrt{x^{2}-1}\right), \\
& G(1)=0, \quad G(2)=\frac{\sqrt{3}}{\sqrt{1+p^{2}}}-\log (2+\sqrt{3}), \\
& G^{\prime}(x)=-\frac{(x-1) f(x)}{2 \sqrt{x^{2}-1}\left(p^{2} x+1-p^{2}\right)^{3 / 2}\left[p^{2} x^{2}+2\left(p^{2} x+1-p^{2}\right)^{3 / 2}+2\left(1-p^{2}\right) x+p^{2}\right]},
\end{aligned}
$$

where $f(x)$ is defined by (2.6).

We divide the discussion into two cases.

Case $1 p=\sqrt{6} / 3$. Then it follows from (2.6) that

$$
f(x)=-\frac{4}{27}(x-1)\left(3 x^{2}+4 x+2\right)<0
$$

for all $x \in(1,2)$.

Therefore,

$$
S_{C A}(a, b)>Q_{\sqrt{6} / 3}(a, b)
$$

for all $a, b>0$ with $a \neq b$ follows easily from (2.21)-(2.24).

Case $2 p=\sqrt{3 / \log ^{2}(2+\sqrt{3})-1}$. Then numerical computations lead to

$$
4 p^{6}+3 p^{4}-4 p^{2}=0.2329 \cdots>0,
$$




$$
\begin{aligned}
& -8 p^{6}+9 p^{4}+4 p^{2}-4=0.6027 \cdots>0, \\
& 3 p^{4}-p^{2}-1=-0.1322 \cdots<0, \\
& f(1)=4\left(3 p^{2}-2\right)=0.7567 \cdots>0, \\
& f(2)=4 p^{6}+11 p^{4}+4 p^{2}-12=-1.669 \cdots<0 .
\end{aligned}
$$

It follows from (2.7) and (2.26)-(2.28) that

$$
\begin{aligned}
f^{\prime}(x) & <-3 p^{4} x^{2}+2\left(4 p^{6}+3 p^{4}-4 p^{2}\right) x^{2}+\left(-8 p^{6}+9 p^{4}+4 p^{2}-4\right) x^{2} \\
& =4\left(3 p^{4}-p^{2}-1\right) x^{2}<0
\end{aligned}
$$

for $x \in(1,2)$.

Equation (2.23) and inequalities (2.29)-(2.31) lead to the conclusion that there exists $\lambda_{2} \in(1,2)$ such that $G(x)$ is strictly decreasing on $\left[0, \lambda_{2}\right]$ and strictly increasing on $\left[\lambda_{2}, 1\right]$.

Note that (2.22) becomes

$$
G(1)=G(2)=0
$$

Therefore,

$$
S_{C A}(a, b)<Q \sqrt{3 / \log ^{2}(2+\sqrt{3})-1}(a, b)
$$

for all $a, b>0$ with $a \neq b$ follows from (2.21) and (2.32) together with the piecewise monotonicity of $G(x)$.

Note that

$$
\begin{aligned}
& \lim _{\mu \rightarrow 0^{+}} \frac{\sqrt{\mu^{2}-\left[\sinh ^{-1}(\mu)\right]^{2}}}{\sinh ^{-1}(\mu) \sqrt{\sqrt{1+\mu^{2}}-1}}=\frac{\sqrt{6}}{3}, \\
& \lim _{\mu \rightarrow \sqrt{3}} \frac{\sqrt{\mu^{2}-\left[\sinh ^{-1}(\mu)\right]^{2}}}{\sinh ^{-1}(\mu) \sqrt{\sqrt{1+\mu^{2}}-1}}=\sqrt{\frac{3}{\log ^{2}(2+\sqrt{3})}-1 .}
\end{aligned}
$$

Therefore, Theorem 2.2 follows from (2.25) and (2.33)-(2.35) together with the fact that inequality (2.20) is equivalent to the inequality (2.36) as follows:

$$
p_{2}<\frac{\sqrt{\mu^{2}-\left[\sinh ^{-1}(\mu)\right]^{2}}}{\sinh ^{-1}(\mu) \sqrt{\sqrt{1+\mu^{2}}-1}}<q_{2} .
$$

Theorem 2.3 Let $p_{3}, q_{3} \in[0,1]$. Then the double inequality

$$
H_{p_{3}}(a, b)<S_{A H}(a, b)<H_{q_{3}}(a, b)
$$

holds for all $a, b>0$ with $a \neq b$ if and only if $p_{3}=1$ and $q_{3} \leq \sqrt{6} / 3$. 
Proof Without loss of generality, we assume that $a>b$. Let $v=(a-b) /(a+b), \lambda=v \sqrt{2-v^{2}}$, $x=\sqrt{1-\lambda^{2}}$ and $p \in[0,1]$. Then $v, \lambda, x \in(0,1)$, and (1.1) and (1.3) lead to

$$
\begin{aligned}
S_{A H}(a, b)-H_{p}(a, b) & =A(a, b)\left[\frac{\lambda}{\tanh ^{-1}(\lambda)}+p^{2}\left(1-\sqrt{1-\lambda^{2}}\right)-1\right] \\
& =\frac{A(a, b)\left[1-p^{2}\left(1-\sqrt{1-\lambda^{2}}\right)\right]}{\tanh ^{-1}(\lambda)} H(x),
\end{aligned}
$$

where

$$
\begin{aligned}
& H(x)=\frac{\sqrt{1-x^{2}}}{p^{2} x+\left(1-p^{2}\right)}-\tanh ^{-1}\left(\sqrt{1-x^{2}}\right), \\
& H(1)=0, \\
& H^{\prime}(x)=-\frac{1-x}{x \sqrt{1-x^{2}}\left[p^{2} x+\left(1-p^{2}\right)\right]^{2}} g(x),
\end{aligned}
$$

where

$$
g(x)=\left(p^{4}+p^{2}-1\right) x-p^{4}+2 p^{2}-1 .
$$

We divide the discussion into two cases.

Case $1 p=\sqrt{6} / 3$. Then (2.41) leads to

$$
g(x)=-\frac{1}{9}(1-x)<0
$$

for $x \in(0,1)$.

Therefore,

$$
S_{A H}(a, b)<H_{\sqrt{6} / 3}(a, b)
$$

for all $a, b>0$ with $a \neq b$ follows easily from (2.38)-(2.40) and (2.42).

Case $2 p=1$. Then it follows from (1.3) and (1.4) that

$$
S_{A H}(a, b)>H(a, b)=H_{1}(a, b)
$$

for all $a, b>0$ with $a \neq b$.

Note that

$$
\begin{aligned}
& \lim _{\lambda \rightarrow 0^{+}} \sqrt{\frac{\tanh ^{-1}(\lambda)-\lambda}{\tanh ^{-1}(\lambda)\left(1-\sqrt{1-\lambda^{2}}\right)}}=\frac{\sqrt{6}}{3}, \\
& \lim _{\lambda \rightarrow 1} \sqrt{\frac{\tanh ^{-1}(\lambda)-\lambda}{\tanh ^{-1}(\lambda)\left(1-\sqrt{1-\lambda^{2}}\right)}}=1 .
\end{aligned}
$$

Therefore, Theorem 2.3 follows from (2.43)-(2.46) and the fact that inequality (2.37) is equivalent to

$$
q_{3}<\sqrt{\frac{\tanh ^{-1}(\lambda)-\lambda}{\tanh ^{-1}(\lambda)\left(1-\sqrt{1-\lambda^{2}}\right)}}<p_{3} .
$$


Theorem 2.4 Let $p_{4}, q_{4} \in[0,1]$. Then the double inequality

$$
C_{p_{4}}(a, b)<S_{A C}(a, b)<C_{q_{4}}(a, b)
$$

holds for all $a, b>0$ with $a \neq b$ if and only if $p_{4} \leq \sqrt{3 \sqrt{3} / \pi-1}$ and $q_{4} \geq \sqrt{6} / 3$.

Proof Without loss of generality, we assume that $a>b$. Let $v=(a-b) /(a+b), \mu=v \sqrt{2+v^{2}}$, $x=\sqrt{1+\mu^{2}}$, and $p \in[0,1]$. Then $v \in(0,1), \mu \in(0, \sqrt{3}), x \in(1,2)$, and (1.2) and (1.3) lead to

$$
\begin{aligned}
S_{A C}(a, b)-C_{p}(a, b) & =A(a, b)\left[\frac{\mu}{\arctan (\mu)}-p^{2}\left(\sqrt{1+\mu^{2}}-1\right)-1\right] \\
& =\frac{A(a, b)\left[1+p^{2}\left(\sqrt{1+\mu^{2}}-1\right)\right]}{\arctan (\mu)} J(x),
\end{aligned}
$$

where

$$
\begin{aligned}
& J(x)=\frac{\sqrt{x^{2}-1}}{p^{2} x+\left(1-p^{2}\right)}-\arctan \left(\sqrt{x^{2}-1}\right), \\
& J(1)=0, \quad J(2)=\frac{\sqrt{3}}{p^{2}+1}-\frac{\pi}{3}, \\
& J^{\prime}(x)=-\frac{x-1}{x \sqrt{x^{2}-1}\left[p^{2} x+\left(1-p^{2}\right)\right]^{2}} g(x),
\end{aligned}
$$

where $g(x)$ is defined by (2.41).

We divide the discussion into two cases.

Case $1 p=\sqrt{6} / 3$. Then (2.41) leads to

$$
g(x)=\frac{1}{9}(x-1)>0
$$

for $x \in(1,2)$.

Therefore,

$$
S_{A C}(a, b)<C_{\sqrt{6} / 3}(a, b)
$$

for all $a, b>0$ with $a \neq b$ follows easily from (2.48)-(2.51).

Case $2 p=\sqrt{3 \sqrt{3} / \pi-1}$. Then numerical computations lead to

$$
\begin{aligned}
& p^{4}+p^{2}-1=\frac{27-\pi^{2}-3 \sqrt{3} \pi}{\pi^{2}}>0, \\
& g(1)=3 p^{2}-2=\frac{9 \sqrt{3}-5 \pi}{\pi}<0, \\
& g(2)=p^{4}+4 p^{2}-3=\frac{27-6 \pi^{2}+6 \sqrt{3} \pi}{\pi^{2}}>0 .
\end{aligned}
$$

From (2.41) and (2.50) together with (2.53)-(2.55) we clearly see that there exists $\lambda_{3} \in$ $(1,2)$ such that $J(x)$ is strictly increasing on $\left[1, \lambda_{3}\right]$ and strictly decreasing on $\left[\lambda_{3}, 2\right]$. 
Note that (2.49) becomes

$$
J(1)=J(2)=0 .
$$

It follows from (2.56) and the piecewise monotonicity of $J(x)$ that

$$
J(x)>0
$$

for all $x \in(1,2)$.

Therefore,

$$
S_{A C}(a, b)>C_{\sqrt{3 \sqrt{3} / \pi-1}}(a, b)
$$

for all $a, b>0$ with $a \neq b$ follows from (2.48) and (2.58).

Note that

$$
\begin{aligned}
& \lim _{\mu \rightarrow 0^{+}} \sqrt{\frac{\mu-\arctan (\mu)}{\arctan (\mu)\left(\sqrt{1+\mu^{2}}-1\right)}}=\frac{\sqrt{6}}{3}, \\
& \lim _{\mu \rightarrow 1} \sqrt{\frac{\mu-\arctan (\mu)}{\arctan (\mu)\left(\sqrt{1+\mu^{2}}-1\right)}}=\sqrt{\frac{3 \sqrt{3}}{\pi}-1 .}
\end{aligned}
$$

Therefore, Theorem 2.4 follows from (2.52) and (2.58)-(2.60) together with the fact that inequality (2.47) is equivalent to

$$
p_{4}<\sqrt{\frac{\mu-\arctan (\mu)}{\arctan (\mu)\left(\sqrt{1+\mu^{2}}-1\right)}}<q_{4} .
$$

\section{Competing interests}

The authors declare that they have no competing interests.

\section{Authors' contributions}

Z-HS provided the main idea and carried out the proof of Theorem 2.1. W-MQ carried out the proof of Theorem 2.2. Y-MC carried out the proof of Theorems 2.3 and 2.4. All authors read and approved the final manuscript.

\section{Author details}

${ }^{1}$ School of Mathematics and Computation Sciences, Hunan City University, Yiyang, 413000, China. ${ }^{2}$ School of Distance Education, Huzhou Broadcast and TV University, Huzhou, 313000, China.

\section{Acknowledgements}

The research was supported by the Natural Science Foundation of China under Grants 61374086 and 11171307, the Natural Science Foundation of the Open University of China under Grant Q1601E-Y and the Natural Science Foundation of Zhejiang Broadcast and TV University under Grant XKT-13Z04.

Received: 12 September 2014 Accepted: 12 November 2014 Published: 26 Nov 2014

\section{References}

1. Neuman, E, Sándor, J: On the Schwab-Borchardt mean. Math. Pannon. 14(2), 253-266 (2003)

2. Neuman, E, Sándor, J: On the Schwab-Borchardt mean II. Math. Pannon. 17(1), 49-59 (2006)

3. Neuman, E: Inequalities for the Schwab-Borchardt mean and their applications. J. Math. Inequal. 5(4), $601-609$ (2011)

4. Neuman, E: On some means derived from the Schwab-Borchardt mean. J. Math. Inequal. 8(1), 171-183 (2014)

5. Neuman, E: On some means derived from the Schwab-Borchardt mean II. J. Math. Inequal. 8(2), 361-370 (2014)

6. Neuman, E: A one-parameter family of bivariate means. J. Math. Inequal. 7(3), 399-412 (2013)

7. He, Z-Y, Chu, Y-M, Wang, M-K: Optimal bounds for Neuman means in terms of harmonic and contraharmonic means. J. Appl. Math. 2013, Article ID 807623 (2013) 
8. Qian, W-M, Chu, Y-M: Optimal bounds for Neuman means in terms of geometric, arithmetic and quadratic means. J. Inequal. Appl. 2014, Article ID 175 (2014)

9. Chu, Y-M, Qian, W-M: Refinements of bounds for Neuman means. Abstr. Appl. Anal. 2014, Article ID 354132 (2014)

10. Neuman, E: Sharp inequalities involving Neuman-Sándor and logarithmic means. J. Math. Inequal. 7(3), 413-419 (2013)

10.1186/1029-242X-2014-468

Cite this article as: Shao et al.: Sharp bounds for Neuman means in terms of one-parameter family of bivariate means. Journal of Inequalities and Applications 2014, 2014:468

Submit your manuscript to a SpringerOpen ${ }^{\circ}$ journal and benefit from:

- Convenient online submission

- Rigorous peer review

- Immediate publication on acceptance

- Open access: articles freely available online

- High visibility within the field

- Retaining the copyright to your article

Submit your next manuscript at $>$ springeropen.com 